Working Paper 09-30

Statistics and Econometrics Series 10

May 2009
Departamento de Estadística Universidad Carlos III de Madrid

Calle Madrid, 126 28903 Getafe (Spain)

Fax (34) 91 624-98-49

\title{
EXACT GOODNESS-OF-FIT TESTS FOR CENSORED DATA
}

\author{
Aurea Grané
}

\begin{abstract}
The statistic introduced in Fortiana and Grané (2003) is modified so that it can be used to test the goodness-of-fit of a censored sample, when the distribution function is fully specified. Exact and asymptotic distributions of three modified versions of this statistic are obtained and exact critical values are given for different sample sizes. Empirical power studies show the good performance of these statistics in detecting symmetrical alternatives.
\end{abstract}

Keywords: Goodness-of-fit, Censored Samples, Maximum Correlation, Exact Distribution, $L$-statistics.

AMS subject classification: 62G10, 62G20, 62G30, 62E15, 62N05.

A. Grané, Statistics Department, Universidad Carlos III de Madrid, C/ Madrid 126, 28903 Getafe (Madrid), Spain, e-mail: aurea.grane@uc3m.es . Corresponding author.

Work partially supported by Spanish grants SEJ2007-64500 and MTM2006-09920 (Ministry of Education and Science- FEDER). 


\title{
Exact goodness-of-fit tests for censored data
}

\author{
Aurea Grané \\ Statistics Department. Universidad Carlos III de Madrid.
}

\begin{abstract}
The statistic introduced in Fortiana and Grané (2003) is modified so that it can be used to test the goodness-of-fit of a censored sample, when the distribution function is fully specified. Exact and asymptotic distributions of three modified versions of this statistic are obtained and exact critical values are given for different sample sizes. Empirical power studies show the good performance of these statistics in detecting symmetrical alternatives.
\end{abstract}

Keywords: Goodness-of-fit, censored samples, maximum correlation, exact distribution, $L$-statistics.

AMS subject classification: $62 \mathrm{G} 10,62 \mathrm{G} 20,62 \mathrm{G} 30,62 \mathrm{E} 15,62 \mathrm{~N} 05$.

\section{Introduction}

The usual way to measure system reliability is to test completed products or components, under conditions that simulate real life, until failure occurs. One may think that the more data available, the more confidence one will have in the reliability level, but this practice is often expensive and time-consuming. Hence, it is of special interest to analyze product life before all test units fail. This situation leads to censored samples, that are encountered naturally in reliability studies. The present paper is concerned on goodness-of-fit tests for this type of data with particular censoring schemes.

Let $y_{1}, \ldots, y_{n}$ be independent and identically distributed (iid) random variables with cumulative distribution function (cdf) $F$ and consider the ordered sample $y_{(1)}<$ $\ldots<y_{(n)}$. In the following we adopt the notation of Stephens and D'Agostino (1986). When some of the observations are missing the sample is said to be censored. If all the observations less than $y_{(s)}(s>1)$ are missing the sample is left-censored and if all the observations greater than $y_{(r)}(r<n)$ are missing, it is right-censored; in either case the sample is said to be singly-censored. If the observations are missing at both ends, the sample is doubly-censored. Censoring may occur for random values of $s$ or $r$ (Type I or time censoring) or for fixed values (Type II or failure censoring).

Research project partially supported by Spanish grants SEJ2007-64500 and MTM2006-09920 (Ministry of Education and Science-FEDER)

Author's address: Statistics Department, Universidad Carlos III de Madrid, c/ Madrid, 126, 28903Getafe, Spain.

E-mail: aurea.grane@uc3m.es

Date: May 6, 2009. 
In Fortiana and Grané (2003) we proposed a goodness-of-fit statistic for complete samples to test the null hypothesis $H_{0}: F(y)=F_{0}(y)$, where $F_{0}(y)$ is a completely specified cdf, or equivalently to test that $x_{1}, \ldots, x_{n}$, where $x_{i}=F_{0}\left(y_{i}\right)$, are iid random variables uniformly distributed in the $[0,1]$ interval. The statistic is based on Hoeffding's maximum correlation (see the definition below) between the empirical cdf and the hypothesized. When there is no censoring, we found out that the test based on the proposed statistic can advantageously replace those of Kolmogorov-Smirnov, Cramér-von Mises and Anderson-Darling for a wide range of alternatives.

Here this statistic is modified so that it can be used to test the goodness-of-fit of a certain proportion of the data, e.g., the sample may be (singly- or doubly-) censored. The paper proceeds as follows: in Section 2 the exact distributions of three modifications of the statistic are deduced and exact critical values are obtained for different sample sizes and different significance levels. In Section 3 we give some conditions under which the convergence to the normal distribution can be asserted. In Section 4 we study the power of the exact tests based on these statistics for five parametric families of alternative distributions with support contained in the $[0,1]$ interval, where we conclude that the tests based on our proposals have a good performance in detecting symmetrical alternatives, whereas the tests based on the Kolmogorov-Smirnov, Cramér-von Mises and Anderson-Darling statistics are biased for some of these alternatives. In Section 5 we give some examples of applicability in the engineering context.

\section{Exact distributions}

We start by introducing Hoeffding's maximum correlation and recall how the $Q_{n}$ statistic is obtained in the case of complete samples.

Let $F_{1}$ and $F_{2}$ be two cdf's with second order moments. Hoeffding's maximum correlation between $F_{1}$ and $F_{2}$, henceforth denoted by $\rho^{+}\left(F_{1}, F_{2}\right)$, is defined as the maximum of the correlation coefficients of bivariate distributions having $F_{1}$ and $F_{2}$ as marginals:

$$
\rho^{+}\left(F_{1}, F_{2}\right)=\frac{1}{\sigma_{1} \sigma_{2}}\left(\int_{0}^{1} F_{1}^{-}(p) F_{2}^{-}(p) d p-\mu_{1} \mu_{2}\right)
$$

where $F_{i}^{-}$is the left-continuous pseudoinverses of $F_{i}, \mu_{i}$ and $\sigma_{i}^{2}$ are, respectively, the expectation and variance of $F_{i}, i=1,2$ (see, e.g., Cambanis, Simons, and Stout 1976). Since $\rho^{+}\left(F_{1}, F_{2}\right)$ equals 1 if and only if $F_{1}=F_{2}$ (almost everywhere) up to a scale and location change, it is a measure of proximity between two distributions and yields a goodness-of-fit test statistic when considering the empirical and hypothesized distributions.

In Fortiana and Grané (2003) we studied the test of uniformity based on

$$
Q_{n}=\frac{s_{n}}{\sqrt{1 / 12}} \rho^{+}\left(F_{n}, F_{U}\right)
$$

where $F_{n}$ is the empirical cdf of $n$ iid real-valued random variables, $s_{n}$ is the sample standard deviation and $F_{U}$ is the cdf of a uniform in $[0,1]$ random variable. The exact distribution of $Q_{n}$ was obtained and its small and large sample properties 
were studied (see also Grané and Tchirina 2009, where local Bahadur asymptotic optimality domains for $Q_{n}$ are obtained).

In the following we deduce the expressions of the modified $Q_{n}$ statistic for singly- and doubly-censored samples and obtain their exact probability density functions (pdf's) under the null hypothesis of uniformity. For all the statistics we give tables of exact critical values for different sample sizes and different significance levels.

\section{$2.1 \quad$ Right-censored samples}

Let $y_{(1)}<\ldots<y_{(n)}$ be the ordered sample. Suppose that the sample is rightcensored of Type I; the $y_{i}$-values are known to be less than a fixed value $y^{*}$. The set of available transformed $x_{i}$-values $\left(x_{i}=F_{0}\left(y_{i}\right)\right)$ is then $x_{(1)}<\ldots<x_{(r)}<t$, where $t=F_{0}\left(y^{*}\right)$. If the censoring is of Type II, there are again $r$ values $x_{(i)}$, with $x_{(r)}$ the largest and $r$ fixed.

Proposition 2.1 Under the null hypothesis of uniformity:

(i) The modified $Q_{n}$ statistic for Type I right-censored data is

$$
{ }_{1} Q_{t n}=\sum_{i=1}^{r+1} a_{i} x_{(i)},
$$

where $a_{i}=6\left((2 i-1)(r+1)-n^{2}\right) /\left(n^{2}(r+1)\right)$, for $1 \leq i \leq r$ and $a_{r+1}=$ $6 r\left(n^{2}-r^{2}-r\right) /\left(n^{2}(r+1)\right)$.

(ii) The modified $Q_{n}$ statistic for Type II right-censored data is

$$
{ }_{2} Q_{r n}=\sum_{i=1}^{r} a_{i} x_{(i)},
$$

where $a_{i}=6\left((2 i-1) r-n^{2}\right) /\left(n^{2} r\right)$, for $1 \leq i \leq r-1$ and $a_{r}=6(r-1)\left(n^{2}-\right.$ $r(r-1)) /\left(n^{2} r\right)$.

Proof:

(i) For type I right-censored data, suppose $t(t<1)$ is the fixed censoring value. This value can be added to the sample set (see Stephens 1986), and the statistic can be calculated by using $x_{(r+1)}=t$. Note that it is possible to have $r=n$ observations less than $t$, since when the value $t$ is added the new sample has size $n+1$.

From formulas (2) and (1) we have that

$$
Q_{n}=12\left(\int_{0}^{1} F_{n}^{-}(p) F_{U}^{-}(p) d p-\frac{1}{2} \bar{x}_{n}\right) .
$$

Noticing that the pseudo-inverse of the empirical cdf is

$$
F_{n}^{-}(p)= \begin{cases}x_{(i)}, & \frac{i-1}{n}<p \leq \frac{i}{n}, \quad 1 \leq i \leq r, \\ x_{(r+1)}, & \frac{r}{n}<p \leq 1,\end{cases}
$$


for $0 \leq p \leq 1$, the first summand of (5) is

$$
\begin{aligned}
\int_{0}^{1} F_{n}^{-}(p) F_{U}^{-}(p) d p & =\sum_{i=1}^{r} \int_{(i-1) / n}^{i / n} x_{(i)} p d p+\sum_{i=r+1}^{n} \int_{(i-1) / n}^{i / n} x_{(r+1)} p d p \\
& =\frac{1}{2 n^{2}} \sum_{i=1}^{r}(2 i-1) x_{(i)}+\frac{1}{2 n^{2}}\left(n^{2}-r^{2}\right) x_{(r+1)}
\end{aligned}
$$

and subtracting the (available) sample mean, the part of (5) between parenthesis is

$$
\frac{1}{2 n^{2}(r+1)} \sum_{i=1}^{r}\left((2 i-1)(r+1)-n^{2}\right) x_{(i)}+\frac{r\left(n^{2}-r^{2}-r\right)}{2 n^{2}(r+1)} x_{(r+1)} .
$$

(ii) For Type II right-censored data the pseudo-inverse of the empirical cdf is

$$
F_{n}^{-}(p)= \begin{cases}x_{(i)}, & \frac{i-1}{n}<p \leq \frac{i}{n}, \quad 1 \leq i \leq r-1, \\ x_{(r)}, & \frac{r-1}{n}<p \leq 1,\end{cases}
$$

for $0 \leq p \leq 1$. Proceeding analogously, the part of (5) between parenthesis is

$$
\int_{0}^{1} F_{n}^{-}(p) F_{U}^{-}(p) d p=\frac{1}{2 n^{2}} \sum_{i=1}^{r-1}(2 i-1) x_{(i)}+\frac{1}{2}\left(1-\frac{(r-1)^{2}}{n^{2}}\right) x_{(r)}
$$

and subtracting the (available) sample mean, the part of (5) between parenthesis is

$$
\frac{1}{2 n^{2} r} \sum_{i=1}^{r-1}\left((2 i-1) r-n^{2}\right) x_{(i)}+\frac{(r-1)}{2 n^{2} r}\left(n^{2}-r(r-1)\right) x_{(r)} .
$$

Under the null hypothesis, ${ }_{1} Q_{t n}$ and ${ }_{2} Q_{r n}$ are linear combinations of selected order statistics from the $[0,1]$-uniform distribution. Therefore their exact probability density functions can be obtained with the following algorithm, proposed by Dwass (1961), Matsunawa (1985) and Ramallingam (1989).

For Type I right-censored data, let

$$
b_{i}=\sum_{l=i}^{r+1} a_{l}=\frac{6}{n^{2}}\left(2 i-i^{2}-1\right)+\frac{6}{r+1}(i-1), \quad i=1,2, \ldots, r+1,
$$

let $k$ be the number of distinct non-zero $b_{i}$ 's, and $\left(\nu_{1}, \ldots, \nu_{k}\right)$ be the corresponding multiplicities of $\left(b_{1}, \ldots, b_{k}\right)$. Defining on $\mathbb{C}$ the functions:

$$
G(s)=\left[\prod_{j=1}^{k}\left(s+\frac{1}{b_{j}}\right)^{\nu_{j}}\right]^{-1}, \quad G_{l}(s)=\left(s+\frac{1}{b_{l}}\right)^{\nu_{l}} G(s), \quad l=1,2, \ldots, k,
$$

the exact pdf of ${ }_{1} Q_{t n}$ statistic, under $H_{0}$, is given by $f_{1} Q_{t n}(s)=$

$$
\sum_{l=1}^{k} \sum_{m=1}^{\nu_{l}} \operatorname{sign}\left(b_{l}\right) C_{l, m}^{\sharp} \chi\left(\frac{s}{b_{l}}\right) \chi\left(1-\frac{s}{b_{l}}\right) s^{m-1}\left(1-\frac{s}{b_{l}}\right)^{n-m} / B(m, n-m+1)
$$


where $\chi(x)$ is the indicator of the interval $[x>0], B(a, b)$ is the Beta function,

$$
C_{l, m}^{\sharp}=\left(\prod_{j=1}^{k}\left(b_{j}\right)^{-\nu_{j}}\right) C_{l, m}, \quad C_{l, m}=\frac{G_{l}^{\left(\nu_{l}-m\right)}\left(-1 / b_{l}\right)}{\left(\nu_{l}-m\right) !},
$$

and $G_{l}^{(j)}$ denotes the $j$-th derivative of $G_{l}$.

Analogously, for Type II right-censored data, the pdf of ${ }_{2} Q_{r n}$ is found by applying the previous algorithm, taking into account that in this case

$$
b_{i}=\sum_{l=i}^{r} a_{l}=\frac{6}{n^{2}}\left(2 i-i^{2}-1\right)+\frac{6}{r}(i-1), \quad i=1,2 \ldots, r .
$$

Remark 1 For left-censored data, note that from the $r$ largest observations one can compute the values $x_{(i)}^{*}=1-x_{(n+1-i)}$, for $i=1, \ldots, r$, so that the sample becomes right-censored. In Type I censoring the left-censoring fixed value converts to $t^{*}=1-t$, to be used as the right-censoring fixed point.

Mathematica programs implementing this algorithm are available from the author. As an illustration of its application, critical values for $5 \%$ and $2.5 \%$ significance levels are computed to test the null hypothesis of uniformity. They are reproduced in Table 1.

Table 1: Lower- and upper-tail critical values of ${ }_{1} Q_{t n}$ and ${ }_{2} Q_{r n}$ for $p$ proportions of

\begin{tabular}{|c|c|c|c|c|c|c|c|c|c|c|c|c|}
\hline & \multicolumn{6}{|c|}{$5 \%$ significance level } & \multicolumn{6}{|c|}{$2.5 \%$ significance level } \\
\hline$p$ & & $=10$ & & $=20$ & & $=30$ & & $=10$ & & $=20$ & $n=$ & $=30$ \\
\hline$\overline{0.3}$ & 0.2221 & 1.4577 & 0.3563 & 1.3467 & 0.4288 & 1.2751 & 0.1692 & 1.6314 & 0.3009 & 1.4778 & 0.3760 & 1.3828 \\
\hline 0.4 & 0.3566 & 1.6455 & 0.5260 & 1.5568 & 0.6133 & 1.4925 & 0.2874 & 1.8059 & 0.4590 & 1.6805 & 0.5514 & 1.5952 \\
\hline 0.5 & 0.4867 & 1.7344 & 0.6820 & 1.6820 & 0.7788 & 1.6315 & 0.4071 & 1.8725 & 0.6088 & 1.7917 & 0.7128 & 1.7239 \\
\hline 0.6 & 0.5980 & 1.7228 & 0.8095 & 1.7167 & 0.9104 & 1.6853 & 0.5142 & 1.8326 & 0.7356 & 1.8076 & 0.8450 & 1.7631 \\
\hline 0.7 & 0.6751 & 1.6095 & 0.8935 & 1.6554 & 0.9937 & 1.6465 & 0.5939 & 1.6875 & 0.8243 & 1.7240 & 0.9335 & 1.7067 \\
\hline 0.8 & 0.6994 & 1.4005 & 0.9172 & 1.4956 & 1.0125 & 1.5103 & 0.6278 & 1.4500 & 0.8580 & 1.5414 & 0.9619 & 1.5516 \\
\hline 0.9 & 0.6337 & 1.1368 & 0.8547 & 1.2518 & 0.9443 & 1.2876 & 0.5789 & 1.1735 & 0.8101 & 1.2817 & 0.9067 & 1.3886 \\
\hline
\end{tabular}
data in the sample.

\begin{tabular}{|c|c|c|c|c|c|c|c|c|c|c|c|c|}
\hline \multirow{3}{*}{$\frac{p}{0.3}$} & \multicolumn{6}{|c|}{$5 \%$ significance level } & \multicolumn{6}{|c|}{$2.5 \%$ significance level } \\
\hline & \multicolumn{2}{|c|}{$n=10$} & \multicolumn{2}{|c|}{$n=20$} & \multicolumn{2}{|c|}{$n=30$} & \multicolumn{2}{|c|}{$n=10$} & \multicolumn{2}{|c|}{$n=20$} & \multicolumn{2}{|c|}{$n=30$} \\
\hline & 0.0999 & 1.1678 & 0.2714 & 1.2105 & 0.3659 & 1.1861 & 0.0684 & 1.3418 & 0.2240 & 1.3423 & 0.3172 & 1.2940 \\
\hline 0.4 & 0.2221 & 1.4577 & 0.4418 & 1.4621 & 0.5531 & 1.4284 & 0.1692 & 1.6314 & 0.3800 & 1.5905 & 0.4938 & 1.5334 \\
\hline 0.5 & 0.3566 & 1.6455 & 0.6066 & 1.6303 & 0.7267 & 1.5943 & 0.2874 & 1.8059 & 0.5359 & 1.7478 & 0.6615 & 1.6906 \\
\hline 0.6 & 0.4867 & 1.7344 & 0.7503 & 1.7110 & 0.8712 & 1.6773 & 0.4071 & 1.8725 & 0.6760 & 1.8119 & 0.8051 & 1.7604 \\
\hline 0.7 & 0.5980 & 1.7228 & 0.8579 & 1.6983 & 0.9722 & 1.6701 & 0.5142 & 1.8326 & 0.7857 & 1.7784 & 0.9098 & 1.7364 \\
\hline 0.8 & 0.6751 & 1.6095 & 0.9141 & 1.5876 & 1.0145 & 1.5666 & 0.5939 & 1.6875 & 0.8493 & 1.6446 & 0.9602 & 1.6141 \\
\hline 0.9 & 0.6994 & 1.4005 & 0.8993 & 1.3818 & 0.9789 & 1.3889 & 0.6278 & 1.4500 & 0.8469 & 1.4180 & 0.9367 & 1.4600 \\
\hline
\end{tabular}

\subsection{Doubly-censored samples}

Let $x_{(s)}<\ldots<x_{(r)}$, with $1<s<r<n$, be the available $x_{i}$-values from a Type II doubly-censored sample. 
Proposition 2.2 Under the null hypothesis of uniformity, the modified $Q_{n}$ statistic for Type II doubly-censored data is

$$
{ }_{2} Q_{s r, n}=\sum_{i=s}^{r} a_{i} x_{(i)},
$$

where $a_{s}=6\left(s-n^{2} /(r-s+1)\right) / n^{2}, a_{i}=6\left((2 i-1)-n^{2} /(r-s+1)\right) / n^{2}$, for $s+1 \leq i \leq r-1$ and $a_{r}=6\left(n^{2}-(r-1)^{2}-n^{2} /(r-s+1)\right) / n^{2}$.

Proof: Since in this case the pseudo-inverse of the empirical cdf is

$$
F_{n}^{-}(p)= \begin{cases}x_{(s)}, & 0<p \leq \frac{s}{n} \\ x_{(i)}, & \frac{i-1}{n}<p \leq \frac{i}{n}, \quad s+1 \leq i \leq r-1 \\ x_{(r)}, & \frac{r-1}{n}<p \leq 1\end{cases}
$$

for $0 \leq p \leq 1$, the first summand of (5) is equal to

$$
\begin{aligned}
\int_{0}^{1} F_{n}^{-}(p) F_{U}^{-}(p) d p & =\sum_{i=1}^{s} \int_{(i-1) / n}^{i / n} x_{(s)} p d p+\sum_{i=s+1}^{r-1} \int_{(i-1) / n}^{i / n} x_{(i)} p d p+\sum_{i=r}^{n} \int_{(i-1) / n}^{i / n} x_{(r)} p d p \\
& =\frac{1}{2 n^{2}} s x_{(s)}+\frac{1}{2 n^{2}} \sum_{i=s+1}^{r-1}(2 i-1) x_{(i)}+\frac{1}{2 n^{2}}\left(n^{2}-(r-1)^{2}\right) x_{(r) .}
\end{aligned}
$$

Subtracting the (available) sample mean, the part of (5) between parenthesis is

$$
\frac{1}{2 n^{2}}\left[\left(s-\frac{n^{2}}{r-s+1}\right) x_{(s)}+\sum_{i=s+1}^{r-1}\left((2 i-1)-\frac{n^{2}}{r-s+1}\right) x_{(i)}+\left(n^{2}-(r-1)^{2}-\frac{n^{2}}{r-s+1}\right) x_{(r)}\right] \text {. }
$$

For Type II doubly-censored data, the pdf of ${ }_{2} Q_{s r, n}$ is found by applying the algorithm described in Section 2.1, taking into account that now

$$
b_{i}=\sum_{l=i}^{r} a_{l}=\left\{\begin{array}{l}
\frac{6}{n^{2}} s(1-s), \quad i=s, \\
\frac{6}{n^{2}}\left(2 i-i^{2}-1\right)+\frac{6}{r-s+1}(i-s), \quad i=s+1 \ldots, r .
\end{array}\right.
$$

Analogously, it is possible to obtain tables of critical values for different values of $r$ and $s$ and different sample sizes. A Mathematica program implementing this algorithm is available from the author. As an illustration of its application, critical values for $5 \%$ and $2.5 \%$ significance levels are computed to test the null hypothesis of uniformity, for symmetric double-censoring, where $p=r / n, q=s / n$ and $p=1-q$. They are reproduced in Table 2 .

Concerning to the Mathematica programs implementing the pdf's of ${ }_{1} Q_{t n},{ }_{2} Q_{r n}$ and ${ }_{2} Q_{s r, n}$, it should be mentioned that the original formula (2.3) of Ramallingam (1989) for the pdf consists on a sum of terms, containing indicator functions of overlapping intervals. There we obtain an alternative expression taking disjoint intervals which saves computational resources in calculating the critical values. If $k$ is the number of distinct non-zero $b_{i}$ 's and we consider these coefficients ordered in ascending order, $b_{(1)}<\ldots<b_{(k)}$, then the pdf is defined by parts over the partition $0<b_{(1)}<\ldots<$ $b_{(k)}$, if all the $b_{i}$ 's are positive or either over the partition $b_{(1)}<\ldots<b_{(k)}$ if there are negative $b_{i}$ 's. In all cases, the support of these pdf's is determined by the interval $\left[\min \left\{0, b_{(1)}\right\}, b_{(k)}\right]$. 
Table 2: Lower- and upper-tail critical values of ${ }_{2} Q_{s r, n}$ for different values of $p=r / n$ and $q=s / n$, where $p=1-q$.

\begin{tabular}{ccccc}
\multicolumn{5}{c}{$n=10$} \\
\hline$p$ & $5 \%$ sig. level & $2.5 \%$ & sig. level \\
\hline 0.80 & 0.4458 & 1.3152 & 0.3798 & 1.3978 \\
0.90 & 0.6994 & 1.4005 & 0.6278 & 1.4500 \\
\hline
\end{tabular}

\begin{tabular}{ccccc}
\multicolumn{6}{c}{$n=20$} \\
\hline$p$ & $5 \%$ sig. level & $2.5 \%$ & sig. level \\
\hline 0.80 & 0.5297 & 1.1458 & 0.4790 & 1.2065 \\
0.85 & 0.6518 & 1.2051 & 0.6006 & 1.2535 \\
0.90 & 0.7627 & 1.2378 & 0.7136 & 1.2750 \\
0.95 & 0.8547 & 1.2518 & 0.8101 & 1.2817 \\
\hline
\end{tabular}

\begin{tabular}{|c|c|c|c|c|}
\hline \multicolumn{5}{|c|}{$n=30$} \\
\hline $\bar{p}$ & $5 \% \mathrm{si}$ & g. level & $2.5 \%$ & ig. level \\
\hline 0.80 & 0.5707 & 1.0752 & 0.5278 & 1.1253 \\
\hline 0.833 & 0.6498 & 1.1195 & 0.6072 & 1.1631 \\
\hline 0.867 & 0.7239 & 1.1519 & 0.6824 & 1.1891 \\
\hline 0.90 & 0.7912 & 1.1736 & 0.7517 & 1.2048 \\
\hline
\end{tabular}

\subsection{Expectation and variance}

In this Section we obtain expressions for the exact expectation and variance of ${ }_{2} Q_{r n}$ and ${ }_{2} Q_{s r, n}$ under the null hypothesis of uniformity. In matrix notation, these statistics can be written as

$$
{ }_{2} Q_{r n}=\mathbf{a}^{\prime} \mathbf{x}_{r}
$$

where $\mathbf{x}_{r}=\left(x_{(1)}, \ldots, x_{(r)}\right)^{\prime}, \mathbf{a}=\left(a_{1}, \ldots, a_{r}\right)^{\prime}$, with $a_{i}=6\left((2 i-1) r-n^{2}\right) /\left(n^{2} r\right)$, for $1 \leq i \leq r-1$ and $a_{r}=6(r-1)\left(n^{2}-r(r-1)\right) /\left(n^{2} r\right)$,

$$
{ }_{2} Q_{s r, n}=\mathbf{a}^{\prime} \mathbf{x}_{s r},
$$

where $\mathbf{x}_{s r}=\left(x_{(s)}, \ldots, x_{(r)}\right)^{\prime}, \mathbf{a}=\left(a_{s}, \ldots, a_{r}\right)^{\prime}$, with $a_{s}=6\left(s-n^{2} /(r-s+1)\right) / n^{2}$, $a_{i}=6\left((2 i-1)-n^{2} /(r-s+1)\right) / n^{2}$, for $s+1 \leq i \leq r-1$ and $a_{r}=6\left(n^{2}-(r-1)^{2}-\right.$ $\left.n^{2} /(r-s+1)\right) / n^{2}$.

It is straightforward to prove that when $s=1$ and $r=n$ the previous statistics coincide with the $Q_{n}$ statistic for complete samples (see Proposition 3 of Fortiana and Grané 2003).

Proposition 2.3 Under the null hypothesis of uniformity the exact expectation and variance of ${ }_{2} Q_{r n}$ are given by

$$
E\left({ }_{2} Q_{r n} \mid H_{0}\right)=\frac{\left(3 n^{2}+r-2 r^{2}\right)(r-1)}{n^{2}(n+1)}, \quad \operatorname{var}\left({ }_{2} Q_{r n} \mid H_{0}\right)=\mathbf{a}^{\prime} \mathbf{C}_{r} \mathbf{a},
$$

where $\mathbf{a}$ is the vector of coefficients of (7) and matrix $\mathbf{C}_{r}=\left(c_{i j}\right)_{1 \leq i, j \leq r}$ is defined by the covariances

$$
c_{i j}=\operatorname{cov}\left(\left(x_{(i)}, x_{(j)}\right) \mid H_{0}\right)=\frac{1}{(n+2)(n+1)^{2}}\{(n+1) \min (i, j)-i j\}, \quad 1 \leq i, j \leq r .
$$

Proposition 2.4 Under the null hypothesis of uniformity the exact expectation and variance of ${ }_{2} Q_{s r, n}$ are given by

$$
\begin{aligned}
E\left({ }_{2} Q_{s r, n} \mid H_{0}\right) & =\frac{1}{n^{2}(n+1)}\left(\left(1+3(n-r)-3 n^{2}-4(n-r)^{2}\right)(n-r)\right. \\
& \left.+\left(3 n^{2}-1\right) r+3 r^{2}-2 r^{3}\right) \\
\operatorname{var}\left({ }_{2} Q_{s r, n} \mid H_{0}\right) & =\mathbf{a}^{\prime} \mathbf{C}_{s r} \mathbf{a}
\end{aligned}
$$


where $\mathbf{a}$ is the vector of coefficients of (8) and matrix $\mathbf{C}_{s r}=\left(c_{i j}\right)_{s \leq i, j \leq r}$, with covariances $c_{i j}$ defined as in Proposition 2.3, but for $s \leq i, j \leq r$.

Proof: (of Propositions 2.3 and 2.4). Formulae for the expectation and variance of $\mathbf{x}_{r}$ under the null can be found in David (1981). Expressions for the expectations and variances of ${ }_{2} Q_{r n}$ and ${ }_{2} Q_{s r, n}$ are obtained from (7) and (8), respectively, after some easy but tedious computations. For example, to get the expectation of ${ }_{2} Q_{r n}$ substitute $E\left(\mathbf{x}_{r} \mid H_{0}\right)=\left(\frac{1}{n+1}, \ldots, \frac{r}{n+1}\right)^{\prime}$ for $\mathbf{x}_{r}$ in formula $(7)$.

\section{$3 \quad$ Asymptotic distributions}

In this Section we give conditions under which the asymptotic normality of ${ }_{1} Q_{t n}$, ${ }_{2} Q_{r n}$ and ${ }_{2} Q_{s r, n}$ can be established. With the same notation of Section 2 and under the null hypothesis of uniformity:

Proposition 3.1 If $r=o(n)$, the statistic ${ }_{2} Q_{r n}$ is asymptotically normally distributed, in the sense of

$$
\sup _{-\infty<t<\infty}\left|P\left({ }_{2} Q_{r n}<t\right)-\Phi_{\mu_{n}, \sigma_{n}}(t)\right| \rightarrow 0, \quad(n \rightarrow \infty),
$$

where $\Phi_{\mu_{n}, \sigma_{n}}$ is the cdf of a normal random variable with expectation and variance

$$
\begin{aligned}
\mu_{n} & =\frac{\left(3 n^{2}+r-2 r^{2}\right)(r-1)}{n^{2}(n+1)}, \\
\sigma_{n}^{2} & =\frac{6(r+1)}{5 n^{4}(n+1)^{2} r}\left(5 n^{4}(2 r-1)-15 n^{2}(r-1) r^{2}+r^{2}\left(6 r^{3}-9 r^{2}+r+1\right)\right) .
\end{aligned}
$$

Proof: The proof is based on Theorem 4.4 of Matsunawa (1985). Since the assumptions of that theorem hold, to assert the convergence of ${ }_{2} Q_{r n}$ to the normal distribution we must prove that

$$
\frac{\max _{1 \leq i \leq r}\left|b_{i}\right|}{(n+1) \sigma_{n}} \rightarrow 0, \quad(n \rightarrow \infty),
$$

where coefficients $b_{i}$ 's were defined as $b_{i}=\frac{6}{n^{2}}\left(2 i-i^{2}-1\right)+\frac{6}{r}(i-1)$ for $i=1,2 \ldots, r$. From formulas (4.6) and (4.7) of Matsunawa (1985), we obtain

$$
\begin{gathered}
\mu_{n}=\frac{1}{n+1} \sum_{i=1}^{r} b_{i}=\frac{\left(3 n^{2}+r-2 r^{2}\right)(r-1)}{n^{2}(n+1)}, \\
\sigma_{n}^{2}=\frac{1}{(n+1)^{2}} \sum_{i=1}^{r} b_{i}^{2}=\frac{6(r+1)\left(5 n^{4}(2 r-1)-15 n^{2}(r-1) r^{2}+r^{2}\left(6 r^{3}-9 r^{2}+r+1\right)\right)}{5 n^{4}(n+1)^{2} r} .
\end{gathered}
$$

If $r=o(n)$, condition (9) is fulfilled, since

$$
\max _{1 \leq i \leq r}\left|b_{i}\right| \leq \max _{1 \leq i \leq r}\left(\frac{6}{n^{2}}(i-1)^{2}+\frac{6}{r}(i-1)\right)=6(r-1)\left(\frac{r-1}{n^{2}}+\frac{1}{r}\right) .
$$


Corollary 3.1 Under the assumptions of Proposition 3.1, an analogous result can be established for the statistic ${ }_{1} Q_{t n}$ with

$$
\begin{aligned}
& \mu_{n}=\left(3 n^{2} r-3 r^{2}-2 r^{3}-r\right) /\left(n^{2}(n+1)\right), \\
& \sigma_{n}^{2}=6 r\left(5 n^{2}(2 r+1)-15 n^{2} r(r+1)^{2}+(r+1)^{2}\left(6 r^{3}+9 r^{2}+r-1\right)\right) /\left(5 n^{4}\left(n^{2}+1\right)(r+1)\right) .
\end{aligned}
$$

Proof: The proof is analogous to that of Proposition 3.1, but taking into account that for Type I right-censored data coefficients $b_{i}$ 's are $b_{i}=\frac{6}{n^{2}}\left(2 i-i^{2}-1\right)+\frac{6}{r+1}(i-1)$ for $i=1,2, \ldots, r+1$.

Proposition 3.2 If $r=o(n)$, the statistic ${ }_{2} Q_{s r, n}$, where $s=n-r$, is asymptotically normally distributed, in the sense of

$$
\sup _{-\infty<t<\infty}\left|P\left({ }_{2} Q_{s r, n}<t\right)-\Phi_{\mu_{n}, \sigma_{n}}(t)\right| \rightarrow 0, \quad(n \rightarrow \infty),
$$

where $\Phi_{\mu_{n}, \sigma_{n}}$ is the cdf of a normal random variable with expectation and variance

$$
\begin{aligned}
\mu_{n}= & \frac{1}{n^{2}(n+1)}\left(\left(1+3(n-r)-3 n^{2}-4(n-r)^{2}\right)(n-r)+\left(3 n^{2}-1\right) r+3 r^{2}-2 r^{3}\right), \\
\sigma_{n}^{2}= & \frac{6}{5 n^{4}(n+1)^{2}(n-2 r+1)}\left(19 n^{6}-4 n^{5}(21+37 r)+10 n^{4}\left(9+44 r+47 r^{2}\right)\right. \\
& -10 n^{3}\left(3+34 r+90 r^{2}+76 r^{3}\right)+n^{2}\left(1+80 r+470 r^{2}+940 r^{3}+640 r^{4}\right) \\
& +2 r\left(1+2 r+5 r^{2}+40 r^{3}+69 r^{4}+18 r^{5}\right)+ \\
& \left.-n\left(1+4 r+60 r^{2}+310 r^{3}+540 r^{4}+258 r^{5}\right)\right) .
\end{aligned}
$$

Proof: To prove the convergence of ${ }_{2} Q_{s r, n}$ to the normal distribution is equivalent to proving that (see Theorem 4.4. of Matsunawa 1985)

$$
\frac{\max _{s \leq i \leq r}\left|b_{i}\right|}{(n+1) \sigma_{n}} \rightarrow 0, \quad(n \rightarrow \infty) .
$$

In the case of doubly-censored samples, coefficients $b_{i}$ 's were defined as

$$
b_{i}=\left\{\begin{array}{l}
\frac{6}{n^{2}} s(1-s), \quad i=s, \\
\frac{6}{n^{2}}\left(2 i-i^{2}-1\right)+\frac{6}{r-s+1}(i-s), \quad i=s+1 \ldots, r .
\end{array}\right.
$$

Introducing $s=n-r$, we have that

$$
\max _{s \leq i \leq r}\left|b_{i}\right|=\max _{s+1 \leq i \leq r}\left|b_{i}\right|=\frac{6}{n^{2}(2 r-n+1)} \max _{s+1 \leq i \leq r}\left|-(i-1)^{2}(2 r-n+1)+n^{2}(i-n+r)\right| .
$$

The function inside the absolute value is a polynomial of second order in $i$ with a negative leading term. If $i$ takes values from 1 to $n-1$, this function is always positive and the maximum is attained at $i^{*}=\left[1+n^{2} /(2(2 r-n+1))\right]$, where [.] denotes the nearest integer. However, since $i$ takes values from $s+1$ to $r$, it is possible that the maximum is attained at a certain $i<i^{*}$. In fact, we have that

$$
\begin{aligned}
& \text { if } r<i^{*} \Rightarrow \max _{s \leq i \leq r}\left|b_{i}\right|=\left|b_{r}\right|<\left|b_{i^{*}}\right|, \\
& \text { if } r \geq i^{*} \Rightarrow \max _{s \leq i \leq r}\left|b_{i}\right|=\left|b_{i^{*}}\right| .
\end{aligned}
$$


In both cases it holds that

$$
\max _{s \leq i \leq r}\left|b_{i}\right| \leq\left|b_{i^{*}}\right|=\frac{3\left(4+5 n^{2}+12 r+8 r^{2}-4 n(2+3 r)\right)}{2(n-2 r+1)^{2}} .
$$

Expressions for the expectation and variance are obtained after applying formulas (4.6) and (4.7) of Matsunawa (1985):

$$
\mu_{n}=\frac{1}{n+1}\left(s b_{s}+\sum_{i=s+1}^{r} b_{i}\right), \quad \sigma_{n}^{2}=\frac{1}{(n+1)^{2}}\left(s b_{s}^{2}+\sum_{i=s+1}^{r} b_{i}^{2}\right) .
$$

Finally condition (10) is fulfilled since $\sigma_{n}^{2}=O(1 / n)$.

Remark 2 Note that in Propositions 3.1 and 3.2 the expectation of the limit distribution is the exact expectation of the statistic. Asymptotic critical values for ${ }_{2} Q_{r n}$ and ${ }_{2} Q_{s r, n}$ can be computed from the limit distribution using the corresponding asymptotic variance given in Propositions 3.1 and 3.2, or either the corresponding exact variance given in Propositions 2.3 and 2.4 (see Theorem 4.3 of Matsunawa 1985). In Table 3 we show the relative error (percentage of its absolute value) with respect to the $5 \%$-exact critical values of ${ }_{2} Q_{r n}$ in either situation, for several $p$ proportions of data in a sample of size $n=30$.

Table 3: Relative error (percentage of its absolute value) of the 5\%-asymptotic critical values of ${ }_{2} Q_{r n}$ computed from the limit distribution using (a) the exact variance of the statistic and (b) the asymptotic variance of the statistic, with respect to the exact critical values, for $p$ proportions of data in a sample of size $n=30$.

\begin{tabular}{ccccc} 
& \multicolumn{2}{c}{ (a) exact } & \multicolumn{2}{c}{ (b) asymp. } \\
\cline { 2 - 5 }$p$ & lower & upper & lower & upper \\
\hline 0.3 & $13.69 \%$ & $3.48 \%$ & $29.73 \%$ & $1.47 \%$ \\
0.4 & $6.73 \%$ & $2.30 \%$ & $22.78 \%$ & $3.91 \%$ \\
0.5 & $3.39 \%$ & $1.46 \%$ & $19.93 \%$ & $6.08 \%$ \\
0.6 & $1.47 \%$ & $0.78 \%$ & $18.76 \%$ & $8.20 \%$ \\
0.7 & $0.25 \%$ & $0.20 \%$ & $18.54 \%$ & $10.45 \%$ \\
0.8 & $0.56 \%$ & $0.32 \%$ & $19.02 \%$ & $13.00 \%$ \\
0.9 & $0.95 \%$ & $0.86 \%$ & $20.19 \%$ & $14.04 \%$ \\
\hline
\end{tabular}

\section{Power study and comparisons}

In this section we study the power of the tests based on ${ }_{2} Q_{r n}$ and ${ }_{2} Q_{s r, n}$ for a set of five parametric families of alternative distributions with support contained in the $[0,1]$ interval. They have been chosen so that either the mean or the variance differs from those of the null distribution, which in each case is obtained for a particular value of the parameter.

A1. Lehmann alternatives. Asymmetric distributions with $\operatorname{cdf} F_{\theta}(x)=x^{\theta}$, for $0 \leq$ $x \leq 1$ and $\theta>0$. 
A2. Centered distributions having a U-shaped pdf, for $\theta \in(0,1)$, or wedge-shaped pdf, for $\theta>1$, whose cdf is given by

$$
F_{\theta}(x)= \begin{cases}\frac{1}{2}(2 x)^{\theta}, & 0 \leq x \leq 1 / 2 \\ 1-\frac{1}{2}(2(1-x))^{\theta}, & 1 / 2 \leq x \leq 1\end{cases}
$$

A3. Compressed uniform alternatives in the $[\theta, 1-\theta]$ interval, for $0 \leq \theta<1 / 2$.

A4. Centered distributions with parabolic pdf $f_{\theta}(x)=1+\theta(6 x(1-x)-1)$, for $0 \leq x \leq 1$ and $-2 \leq \theta \leq 1$.

A5. Centered distributions with pdf given by

$$
f_{\theta}(x)=\frac{\sqrt{6 \theta}}{\sqrt{\pi} e^{\theta / 2} \operatorname{erf}(\sqrt{3 \theta / 2})} \exp \{\theta(6 x(1-x)-1)\},
$$

for $0 \leq x \leq 1$ and $\theta>0$, where $\operatorname{erf}(x)=\frac{2}{\sqrt{\pi}} \int_{0}^{x} e^{-t^{2}} d t$ is the error function.

The power of the tests increases with the proportion of data in the sample. To illustrate this finding in Figure 1, we depicted the power functions of the $5 \%$ significance level test based on ${ }_{2} Q_{r n}$, for $p=0.4,0.6,0.8$ proportions of data in the sample. For each value of the parameter, the power was estimated from $N=1000$ simulated samples of size $n=10$ from alternatives A1-A3 as the relative frequency of values of the statistic in the critical region. We used the exact critical values listed in Table 1.

Figure 1: Power functions of the $5 \%$ significance level test based on ${ }_{2} Q_{r n}$, for different $p$ proportions of data in samples of size $n=10$, for (a) A1 alternative, (b) A2 alternative and (d) A3 alternative.

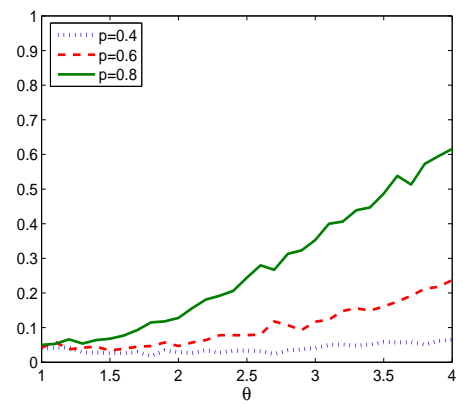

(a)

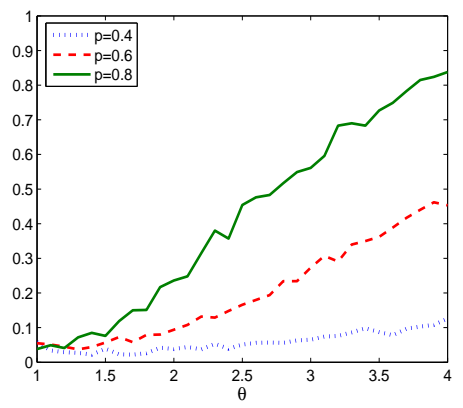

(b)

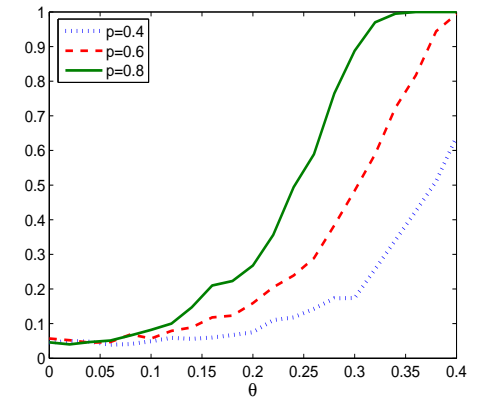

(c)

We have compared the power of the test based on ${ }_{2} Q_{r n}$ with those based on classical statistics such as the Kolmogorov-Smirnov, Cramér-von Mises and Anderson-Darling. Modified versions of these statistics for censored samples, as well as their critical values, can be found in Barr and Davidson (1973), Pettitt and Stephens (1976) and also in Stephens (1986). Figure 2 contains the power functions obtained from $N=1000$ Type II right-censored samples of size $n=25$ and a proportion of data of $p=0.8$ for 
A1-A5 alternatives. We have denoted by ${ }_{2} D_{r n},{ }_{2} W_{r n}^{2}$ and ${ }_{2} A_{r n}^{2}$ the modified versions of the Kolmogorov-Smirnov, Cramér-von Mises and Anderson-Darling statistics, respectively. Sample size and data proportion values of $n=25$ and $p=0.8$ were chosen so that the critical values reproduced in Stephens (1986) were appropriate for comparison. For the test based on ${ }_{2} Q_{r n}$ we computed the exact critical regions. From Figure 2 we can observe the good performance of the ${ }_{2} Q_{r n}$ statistic in detecting symmetrical alternatives.

Figure 2: Power functions of the $5 \%$ significance level tests based on ${ }_{2} Q_{r n},{ }_{2} D_{r n}$, ${ }_{2} W_{r n}^{2}$ and ${ }_{2} A_{r n}^{2}$ for a $p=0.8$ proportion of data in a sample of size $n=25$, for (a) A1 alternative, (b)-(c) A2 alternative, (d) A3 alternative, (e) A4 alternative and (f) A5 alternative.

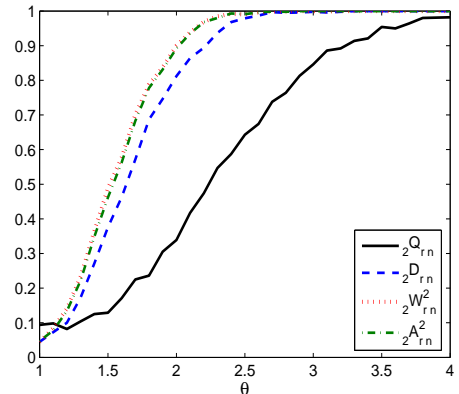

(a)

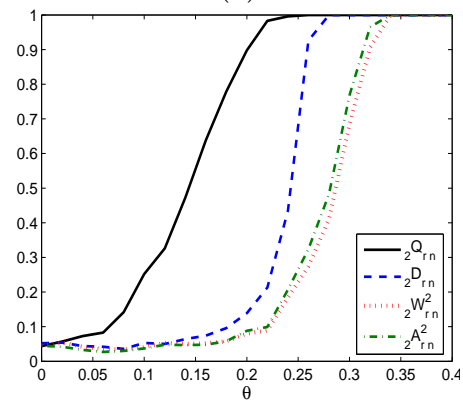

(d)

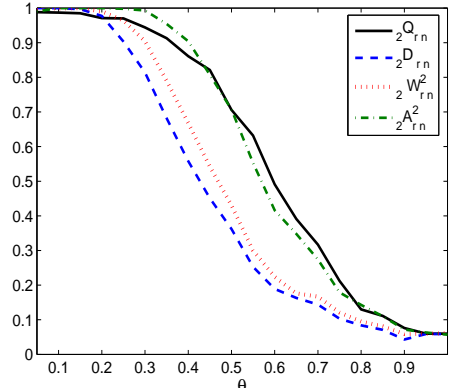

(b)

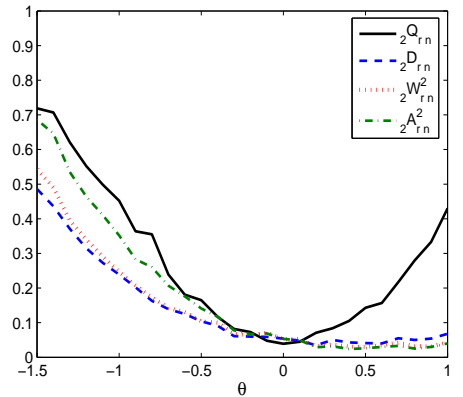

(e)

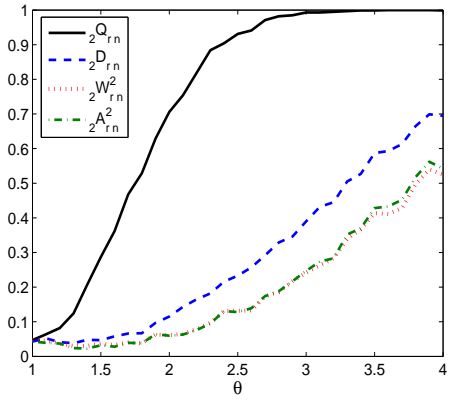

(c)

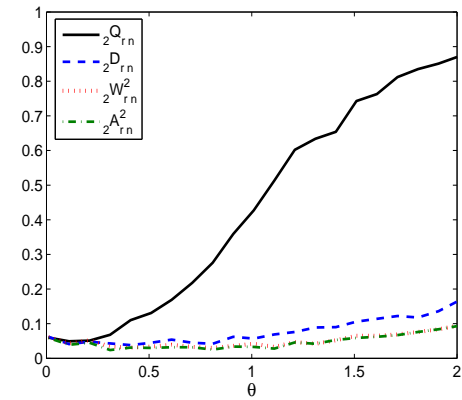

(f)

We also compared the power of the test based on ${ }_{2} Q_{s r, n}$ with those based on the Cramér-von Mises and Anderson-Darling statistics. Critical values for the modified versions of these statistics for doubly-censored samples were computed by Pettitt and Stephens (1976). Figure 3 contains the power functions obtained from $N=1000$ Type II doubly-censored samples of size $n=20$ and $p=r / n=0.9(q=1-p, s=q / n)$ for A1-A5 alternatives. We have denoted by ${ }_{2} W_{r s, n}^{2}$ and ${ }_{2} A_{r s, n}^{2}$ the modified versions of the Cramér-von Mises and Anderson-Darling statistics, respectively. For the test based on ${ }_{2} Q_{s r, n}$ we computed the exact critical regions, whereas for ${ }_{2} W_{r s, n}^{2}$ and ${ }_{2} A_{r s, n}^{2}$ we considered the asymptotical ones, since Pettitt and Stephens (1976) concluded that the distributions of these statistics converge quickly to the asymptotical ones. From Figure 3 we can observe the good performance of the ${ }_{2} Q_{s r, n}$ statistic in detecting symmetrical alternatives. Note also that the modified versions of the Cramér-von 
Mises and Anderson-Darling statistics are biased for A2-A5.

Figure 3: Power functions of the $5 \%$ significance level tests based on ${ }_{2} Q_{s r, n},{ }_{2} W_{s r, n}^{2}$ and ${ }_{2} A_{s r, n}^{2}$ for $n=20$ and $p=0.9$, for (a) A1 alternative, (b) A2 alternative, (c) A3 alternative, (d) A4 alternative and (e) A5 alternative.

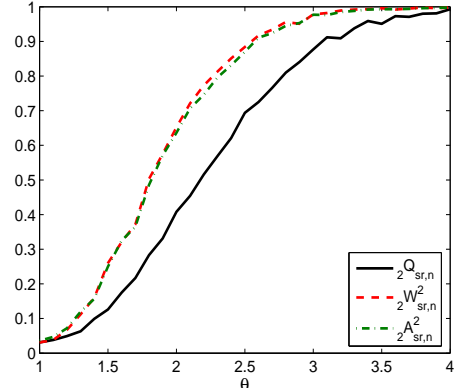

(a)

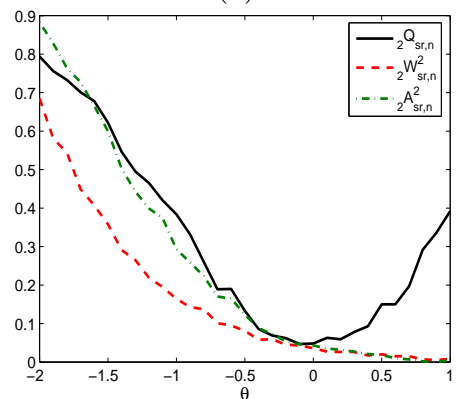

(d)

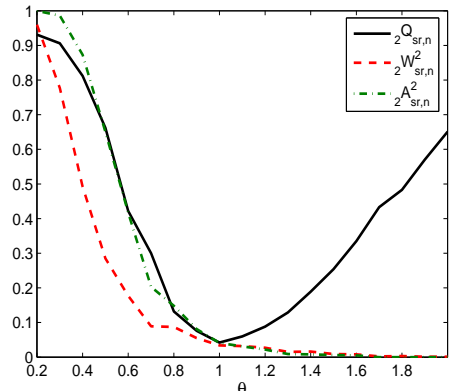

(b)

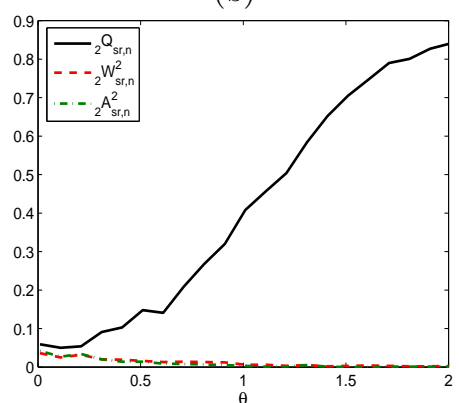

(e)

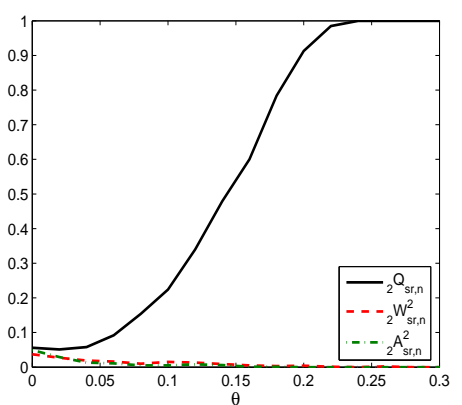

(c)

\section{Data analysis and applications}

In this section we perform goodness-of-fit tests based on ${ }_{1} Q_{t n}$ and ${ }_{2} Q_{r n}$, to show their applicability for, respectively, Type I and Type II right-censored data coming from reliability analysis in engineering.

Data Set 1 Example 5.2.4 of Lawless (1982): Number of thousand miles at which different locomotive controls failed in a life test involving 96 controls. The test was terminated after 135,000 miles, at which time 37 failures had occurred. The failure times for the 37 failed items are: $22.5,37.5,46.0,48.5,51.5,53.0,54.5,57.5,66.5$, $68.0,69.5,76.5,77.0,78.5,80.0,81.5,82.0,83.0,84.0,91.5,93.5,102.5,107.0,108.5$, 112.5, 113.5, 116.0, 117.0, 118.5, 119.0, 120.0, 122.5, 123.0, 127.5, 131.0, 132.5, 134.0. It is concluded that the lognormal distribution provides a reasonable fit for this data.

The hypothesized null distribution is the lognormal $F_{0}(y)=\Phi((\log (y)-5) / 0.7)$, $y>0$, where $\Phi$ is the cdf of the standard normal distribution, with censoring value at $y^{*}=135.0$. After transforming the data with $x_{i}=F_{0}\left(y_{i}\right)$, for $i=1, \ldots, 37$, and the censoring value $t=F_{0}\left(y^{*}\right)=0.4462$, we compute the test statistic (3) and obtain 
${ }_{1} Q_{t n}=1.1127$. We cannot reject the hypothesis of lognormality for a $5 \%$ significance level, since the exact critical values are $c v_{0.025}=0.734049$ and $c v_{0.975}=1.356186$.

Data Set 2 Mann and Fertig (1973) give failure times of aircraft components subjected to life test. Thirteen components were placed on test, but the test was terminated at the time of the tenth failure. Failure times of the 10 components that failed were $0.22,0.50,0.88,1.00,1.32,1.33,1.54,1.76,2.50,3.00$. Engineering considerations indicated that the data should come from a Weibull distribution.

In this example we have Type II right-censoring data with $n=13$ and $r=10$ and the hypothesized null distribution is a Weibull with scale parameter $\lambda=0.5$ and shape parameter $\beta=1.5$. Transforming the data we get $0.0358,0.1175,0.2531,0.2978$, $0.4150,0.4186,0.4912,0.5620,0.7528,0.8407$, and computing the test statistic (4) we obtain ${ }_{2} Q_{r n}=1.5250$. For a $5 \%$ significance level, the exact critical values are $c v_{0.025}=0.682397$ and $c v_{0.975}=1.728394$. Hence we agree that the data may follow a Weibull distribution.

Other applications The test based on ${ }_{2} Q_{r n}$ turns out to be useful in detecting the Weibull family of distributions from the standard exponential. This fact is specially interesting since the Weibull distribution is perhaps the most widely used lifetime distribution model. We illustrate this behavior in Figure 4, where we depict the power functions obtained from $N=1000$ Type II right-censored samples of size $n=25$ and $p=0.8$ to test the null hypothesis of standard exponentiality versus the alternative of a Weibull distribution with scale parameter $\lambda=1$ and shape parameter $\theta>0$.

Figure 4: Power functions of the 5\% significance level goodness-of-fit tests based on ${ }_{2} Q_{r n}{ }_{2} D_{r n},{ }_{2} W_{r n}^{2}$ and ${ }_{2} A_{r n}^{2}$ for a $p=0.8$ proportion of data in a sample of size $n=25$, to detect the Weibull alternative from the standard exponential.

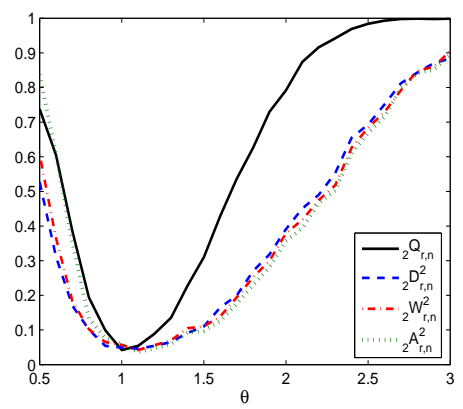

\section{Concluding remarks}

We adapt the goodness-of-fit test based on $Q_{n}$, introduced in Fortiana and Grané (2003), for censored samples. We give tables of exact critical values for different sample sizes and significance levels making these tests easy to implement. The tests based on the modifications of $Q_{n}$ are consistent for all the families of alternatives 
studied, and are more powerful than those based on classical statistics, such as the Kolmogorov-Smirnov, Cramér-von Mises and Anderson-Darling statistics in detecting symmetrical alternatives.

\section{References}

Barr, D. R. and T. Davidson (1973). A Kolmogorov-Smirnov test for censored samples. Technometrics 15, 739-757.

Cambanis, S., G. Simons, and W. Stout (1976). Inequalities for $\mathcal{E} k(x, y)$ when the marginals are fixed. Zeitschrift für Wahrscheinlichkeitstheorie und verwandte Gebiete 36, 285-294.

David, H. A. (1981). Order statistics (2nd ed.). New York: John Willey \& Sons, Inc.

Dwass, M. (1961). The distribution of linear combinations of random divisions of an interval. Trabajos de Estadística e Investigación Operativa 12, 11-17.

Fortiana, J. and A. Grané (2003). Goodness-of-fit tests based on maximum correlations and their orthogonal decompositions. Journal of the Royal Statistical Society B 65 (1), 115-126.

Grané, A. and A. Tchirina (2009). Asymptotic properties of a goodness-of-fit test based on maximum correlations. Working Paper 08-042, Universidad Carlos III de Madrid. (http://hdl.handle.net/10016/3073).

Lawless, J. F. (1982). Statistical Models and Methods for lifetime data. Wiley Series in Probability and Mathematical Statiatics. New York: John Wiley \& Sons.

Mann, N. R. and K. W. Fertig (1973). Tables for obtaining confidence bounds and tolerzance bounds based on best linear invariant estimates of parameters of the extreme value distribution. Technometrics 17, 361-368.

Matsunawa, T. (1985). The exact and approximate distributions of linear combinations of selected order statistics from a uniform distribution. Annals of the Institute of Statistical Mathematics 37, 1-16.

Pettitt, A. N. and M. A. Stephens (1976). Modified Cramér-von Mises statistics for censored data. Biometrika 63 (2), 291-298.

Ramallingam, T. (1989). Symbolic computing the exact distributions of Lstatistics from a uniform distribution. Annals of the Institute of Statistical Mathematics 41, 677-681.

Stephens, M. A. (1986). Tests based on EDF statistics. See Stephens and D'Agostino (1986), pp. 97-193.

Stephens, M. A. and R. B. D'Agostino (Eds.) (1986). Goodness-of-fit Techniques, New York. Marcel Dekker, Inc. 